Doctrina

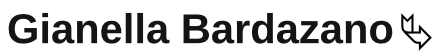

\section{El punto de vista interno y las razones para la acción}

\section{The Internal Point of View and the Reasons for Action \\ O ponto de vista interno e as razões para a ação}

Resumen: El artículo pretende dar cuenta de la importancia del punto de vista interno para la comprensión del concepto de derecho, teniendo en cuenta el distanciamiento de Raz respecto de Hart en este punto, al que le concede primacía conceptual. En ese sentido, para el análisis conceptual de los sistemas jurídicos, el privilegio epistémico conferido al punto de vista interno se articula con la concepción de las reglas como razones excluyentes.

Palabras clave: positivismo, punto de vista interno, reglas, razones excluyentes.

Abstract: The article intends to account for the importance of internal point of view to understand the concept of law, having into account Raz's distancing regarding Hart on this issue, to whom he concedes conceptual primacy. In this sense, for the legal systems' conceptual analysis, the epistemic privilege conferred to internal point of view articulates with the conception of rules as exclusive reasons.

Keywords: Positivism, Internal Point of View, Rules, Exclusive Reasons.

Resumo: $O$ artigo pretende dar conta da importância do ponto de vista interno para a compreensão do conceito de direito, tendo em conta o distanciamento de Raz a respeito de Hart nesse tema, ao qual concede primazia conceitual. Nesse sentido, para a análise conceitual dos sistemas jurídicos, o privilégio epistêmico conferido ao ponto de vista interno se articula com a concepção das regras como razões excludentes.

Palavras-chave: positivismo, ponto de vista interno, regras, razões excludentes.

Recibido: 20190611

Aceptado: 20190702

\footnotetext{
$\stackrel{\leftrightarrow}{\rightarrow}$ Profesora de Filosofía del Derecho, Facultad de Derecho, Universidad de la República. ORCID: 0000-0002-7477-1305

$\triangle$ gbardazano@gmail.com
} 


\section{Sobre la existencia del derecho}

\subsection{Antecedentes. Evolución de la respuesta positivista}

La respuesta teórica de Austin y Bentham a la pregunta por las condiciones de existencia de derecho en una comunidad determinada se basa en atribuirle relevancia a la existencia de un soberano que posee el poder último en la comunidad y cuyos mandatos (órdenes generales dirigidas a clases de acciones e individuos, cuyo cumplimiento es respaldado por la amenaza de sanciones) son obedecidos de modo habitual por los habitantes de la comunidad. En este modelo imperativo simple, un sistema jurídico podría ser identificado a través de hechos tales como los de mandar y obedecer, sin recurrir al contenido de los mandatos y con independencia de la legitimidad del soberano (Vilajosana, 2007, 31). Entre las críticas que ha recibido la teoría imperativa se ubica la de Hart (1961) relativa al escaso rendimiento explicativo de la noción de “orden con amenaza de sanción” para dar cuenta de todos los tipos de disposiciones relevantes en el ámbito jurídico (las reglas de cambio, las reglas de adjudicación y las reglas de reconocimiento). A su vez, la teoría imperativa resulta insuficiente en tanto no distingue el hábito de obediencia del seguimiento de reglas $^{(1)}$ y es por ello que no da cuenta del carácter normativo del derecho. Esta última objeción parece respaldarse, como se verá, en la introducción de la regla de reconocimiento concebida como convención ${ }^{(2)}$.

\subsection{La regla de reconocimiento}

\subsubsection{Características}

La sofisticación del modelo imperativo requiere, señala Josep Vilajosana (2007, 34-37), tener en cuenta la distinción, dentro de los hechos sociales (aquellos cuya existencia depende de la existencia de estados intencionales ${ }^{(3)}$ ), entre hechos no convencionales y convencionales. La diferenciación se vincula a la comprensión de la noción de hecho social en sentido general y en sentido particular, respectivamente. En sentido general, la expresión refiere a comportamientos, actitudes y creencias de los integrantes de una sociedad. En sentido particular, se emplea para una sub- 
clase de dichos comportamientos, actitudes y creencias que se caracteriza por la presencia, entre otros rasgos, de creencias mutuas, intencionalidad colectiva o conocimiento mutuo (Vilajosana, 2007, 35). Precisamente, la insuficiencia de una explicación del derecho que lo reduzca a hábitos de obediencia para dar cuenta del carácter normativo del sistema jurídico, por un lado, y, por otro, la vinculación del punto de vista del participante de la práctica con la noción de creencia mutua o conocimiento mutuo, requieren incorporar en el análisis de las condiciones de existencia de un sistema jurídico lo relativo a los hechos sociales convencionales (hechos sociales en sentido particular) (Vilajosana, 2007, 35).

Las dos condiciones de existencia de un sistema jurídico son, para Hart (1961, 145), en primer lugar, la existencia de una regla de reconocimiento que permita conocer cuáles son los criterios de pertenencia de las demás reglas al sistema jurídico $\mathrm{y}$, en segundo lugar, que las reglas que sean identificadas a partir de la de reconocimiento sean eficaces (Vilajosana, 2007, 37). Con relación a la regla de reconocimiento, es importante recordar que Hart afirma que esta existe como una práctica compleja y normalmente concordante de funcionarios y particulares a la hora de identificar cuál es el derecho en una comunidad determinada, y que su contenido se manifiesta por el uso que esas personas hacen de los criterios de identificación de las fuentes del derecho ${ }^{(4)}$ (Hart, 1961, 137). De ese modo, el uso de criterios compartidos para la identificación del derecho presupone una determinada actitud: el punto de vista interno.

Las autoridades de una determinada sociedad, y entre ellas especialmente los jueces, se comportan de una manera que es consistente con el hecho de seguir la regla que permite identificar el derecho válido de esa sociedad. Ello se refleja en un conjunto de compromisos normativos que aprueban la conducta convergente como justificada y que condenan las desviaciones (Vilajosana 2007, 38).

Esto supone adoptar una versión convencionalista de la primera condición de existencia de un sistema jurídico. En ese sentido, Hart (1961, 105-106) postula la necesidad de una regla de cierre del sistema que confiera validez a las demás reglas pero de la cual no tenga sentido predicar su validez. De ahí que la existencia de la regla de reconocimiento sea una cuestión de hecho, al no poder ser derivada de otras reglas. Se trata, además, de un hecho convencional, en la medida en que presupone una práctica ${ }^{(5)}$ coordinada de identificación del derecho. 
Son las acciones de identificación del derecho por parte de los juristas y las creencias y actitudes mutuas o recíprocas asociadas a ellas las que generan la regla de reconocimiento y, a través de ella, contribuyen a la existencia del derecho de una sociedad (Vilajosana, 2007, 60).

La regla de reconocimiento como criterio para determinar la validez de las demás reglas del sistema jurídico tiene que ser considerada desde el punto de vista interno como un criterio común y público para decidir los casos judiciales concretos. Supone la aceptación de criterios de validez jurídica comunes o compartidos por los funcionarios del sistema (Hart, 1961, 144-145).

Ahora bien, teniendo en cuenta el carácter convencional constitutivo de la regla de reconocimiento, es necesario agregar algunas consideraciones acerca de la normatividad del derecho. Si bien cada regla de reconocimiento refleja o da cuenta de las circunstancias históricas y las convicciones políticas de la comunidad en cuestión ${ }^{(6)}$, cada juez puede emplear los criterios de identificación sea por razones morales, estratégicas, políticas — entre otras-, pero todos deben coincidir en emplear esos criterios y no otros, porque los demás emplean esos y no otros. En otras palabras, la cuestión de la normatividad no se responde

...indagando acerca de si los jueces deben seguir o no una determinada regla de reconocimiento (...). Está claro que esta cuestión solo puede ser contestada acudiendo a un punto de vista moral o político (...). La existencia de una práctica social, en sí misma, por tanto, no supone una obligación moral ni política de comprometerse en dicha práctica. Una regla de reconocimiento solo define cuál es la práctica (...). Pero hay que añadir a continuación: una vez uno se compromete en esa práctica, jugando el papel de juez, existe la obligación jurídica definida por las reglas del juego (Vilajosana, 2007, 45).

En cuanto a la segunda condición de existencia de un sistema jurídico, corresponde señalar que la eficacia es un concepto gradual: una norma puede ser más o menos eficaz según el grado de cumplimiento que obtenga, esto es, dependiendo de la amplitud del conjunto de personas que la obedezcan en relación con el conjunto de destinatarios y de la cantidad de actos de obediencia que genere (Vilajosana, 2007, 53). Puede distinguirse la eficacia normativa (los destinatarios se comportan de acuerdo con lo dispuesto por la norma y es irrelevante conocer los motivos de su comportamiento) de la eficacia causal (la norma ha motivado el comportamiento, es la razón del acto de cumplimiento). En tanto no es posible una sociedad con un 
sistema jurídico cuyas normas no tengan una eficacia causal, una de las condiciones de existencia de un sistema jurídico es la existencia de una autoridad normativa efectiva. La autoridad normativa, como clase de autoridad práctica, implica la pretensión de ser obedecido. El deber de obediencia a las normas que emanan de la autoridad no implica meramente comportarse como la autoridad ordena, sino hacerlo porque la autoridad lo ha ordenado. En ese sentido, las normas jurídicas constituyen razones para la acción, independientemente de su contenido. La existencia de un sistema jurídico requiere una autoridad efectiva y, a su vez, la existencia de una autoridad efectiva requiere un cumplimiento generalizado de sus normas porque las ha dictado la autoridad (requiere eficacia causal de las normas jurídicas) (Vilajosana, 2007, 56). Ahora bien, sostiene Raz que los participantes aceptan el derecho en virtud de consideraciones morales, en la medida en que - de acuerdo con nuestro concepto de derecho - toda imposición de un deber a un tercero (aplicar una regla) no admite otra justificación que no tenga como base una razón moral (Gaido, 2010, 156-157).

Los jueces que aceptan la regla de reconocimiento aceptan una regla que les requiere aceptar otras reglas que imponen obligaciones a otras personas. Ellos, por lo tanto, aceptan una regla que solo puede ser aceptada de buena fe por razones morales (Raz, 1984, 130).

Ellos, por lo tanto, o bien aceptan la regla de reconocimiento por razones morales o, al menos, se comportan como si lo hicieran.

\subsubsection{Raz sobre la regla de reconocimiento}

Raz resume la doctrina de la regla de reconocimiento hartiana en seis postulados, la mayoría de los cuales acepta. Esos son: 1) una regla de reconocimiento exige a los funcionarios que apliquen reglas identificadas a través de los criterios de validez incluidos en ella; 2) todo sistema jurídico tiene al menos una regla de reconocimiento ${ }^{0}$; 3) ningún sistema jurídico tiene más de una regla de reconocimiento; 4) toda regla de reconocimiento es aceptada y practicada por los funcionarios del sistema al que la regla pertenece; 5) pero no es necesario que los funcionarios aprueben la regla de reconocimiento como una regla moralmente buena o justificada; 6) un sistema jurídico consiste en su regla de reconocimiento y en todas las reglas identificadas mediante ella. 
La discrepancia de Raz es fundamentalmente sobre las características 3 y 6 . Con relación a la existencia de más de una regla de reconocimiento ${ }^{(8)}$, señala que no hay ninguna razón para creer que normas válidas que pertenezcan al mismo sistema no puedan estar en conflicto, por lo que, aunque todo sistema jurídico debe contener al menos una regla de reconocimiento, puede de hecho contener más de una. Ahora bien, si el sistema está vigente, las reglas de reconocimiento son reglas consuetudinarias practicadas por los órganos primarios del sistema. De todos modos, que los órganos primarios sigan y apliquen las reglas de reconocimiento no implica que consideren que estas estén moralmente justificadas. Es normal que los sujetos de un sistema institucionalizado entiendan que este está moralmente justificado pero que esos hechos, si bien pueden ser comunes, no son lógicamente necesarios. Que una persona siga una regla exige solo que la considere válida, esto es, que crea que los sujetos normativos están justificados a seguirla, “justificados, quizá, solo porque esa regla ya existe y es practicada y a pesar del hecho de que no debiera haber sido hecha y de que incluso debiera ahora ser cambiada” (Raz, 1991, 170). También el funcionario puede seguir la regla sin tener ninguna creencia sobre por qué está justificado al hacerlo así, o por razones prudenciales (su manera de evitarse una situación social difícil, por ejemplo), o por razones morales que se basen en su rechazo moral del sistema ${ }^{(9)}$. Con relación al postulado 6, señala Raz que, aunque es verdad que los sistemas jurídicos contienen todas las reglas de reconocimiento que se aplican a sus órganos primarios ${ }^{(10)}$ y todas las reglas que aquellas exigen que apliquen los órganos primarios, dichos sistemas pueden contener otras reglas. Las normas que constituyen un sistema institucionalizado incluyen, en primer lugar, las normas dirigidas a los órganos primarios y, en segundo lugar, las dirigidas a los individuos ordinarios (las cuales, por medio de las dirigidas a los órganos primarios, se exige que éstos apliquen). Este segundo tipo de normas son las que se identifican mediante las reglas de reconocimiento. El primer tipo incluye a las reglas de reconocimiento pero puede también incluir otras normas dirigidas a los órganos primarios. La limitación es que si el sistema en cuestión está vigente esas reglas no solo deben estar dirigidas a los órganos primarios sino que deben ser seguidas por ellos (Raz, 1991, 171).

\subsection{El punto de vista interno y el punto de vista externo}

En la elucidación del concepto de derecho la perspectiva del participante tiene privilegio o primacía conceptual; es decir, que no solo sería necesaria la existencia de participantes de la práctica jurídica para la existencia del derecho, sino que es su perspectiva la vía de acceso a la comprensión de la naturaleza del derecho (Gaido, 
2010, 149). Esta posición en cuanto a la prioridad del punto de vista interno en nuestra comprensión de la naturaleza del derecho lo aleja de Hart, en la medida en que si bien Hart considera que la perspectiva del participante es relevante en la explicación del concepto de derecho $(1961,111)$ no asume dicha perspectiva como la perspectiva teórica. Justamente, es en la observación externa de los casos paradigmáticos o centrales de derecho (unión de reglas primarias y secundarias) donde apoya Hart su explicación del concepto de derecho. En ese sentido, los rasgos definitorios del derecho son determinados por el teórico con independencia de los esquemas conceptuales con los cuales están comprometidos los participantes de la práctica jurídica.

El punto de vista interno es el de los sujetos que aceptan o "entienden el derecho como fuente justificatoria de comportamientos" (Gaido, 2010, 149), es decir, como guías de conducta o razones para actuar. En tanto el punto de vista externo es el los sujetos que se ocupan de las reglas como meros observadores que no las aceptan como vinculantes (Hart, 1961, 110).

Hay, entonces, dos puntos de vista teóricos diferentes con relación al derecho. Por un lado, la teoría puede pretender describir el comportamiento social referido al seguimiento de reglas sin recurrir a las creencias y actitudes de aquellos cuyo comportamiento está sujeto a los requerimientos de las instituciones legales. Ante la falta de la información que proveen esas creencias y actitudes, el observador se conforma con registrar regularidades en el comportamiento de una comunidad determinada en relación con la aplicación de sanciones. Esta perspectiva teórica coincide con el denominado punto de vista externo extremo. En contraste, la teoría puede pretender describir el comportamiento social teniendo en cuenta las actitudes de los miembros del grupo. Este punto de vista "hermenéutico" o "no comprometido" busca describir el derecho pero otorgándole relevancia al punto de vista interno (Shapiro, 2006, 1160). Ahora bien, una distinción considerada fundamental en la teoría de Hart es entre el punto de vista práctico y el punto de vista teórico. El primero es aquel que posee el participante que debe decidir cómo va a responder a las exigencias del derecho. El segundo es el del observador que, muchas veces (aunque no necesariamente), es un outsider que estudia el comportamiento de un grupo con relación al derecho. De este modo, respecto del punto de vista práctico son posibles dos actitudes por parte del participante con relación a las reglas: aceptación o no aceptación. Quien acepta las reglas adopta el punto de vista interno. Quien no las acepta $^{(11)}$ adopta el punto de vista externo (Shapiro, 2006, 1160). La aceptación consiste en la disposición de los individuos para adoptar las reglas como guía para 
la propia conducta y también como estándares para la evaluación y crítica de la conducta de otros.

Volviendo al punto de la regla de reconocimiento, en la teoría de Hart, su existencia se muestra como una práctica, esto es: en la manera en que las reglas particulares son identificadas, ya por los tribunales y funcionarios en general, ya por los particulares o sus consejeros (Hart, 1961, 127). El uso por los tribunales de la regla de reconocimiento para identificar reglas particulares del sistema jurídico es lo que caracteriza al punto de vista interno, en la medida en que el uso pone de manifiesto la aceptación de la regla como pauta orientadora. Los enunciados internos constituyen, entonces, enunciados de validez, en tanto que los enunciados externos constituyen enunciados fácticos, que forman parte del lenguaje de un observador externo del sistema que — sin aceptar la regla de reconocimiento — enuncia el hecho de que otros la aceptan (Hart, 1961, 128).

De acuerdo con Hart, los enunciados externos pueden ser de dos tipos, según el observador - sin aceptar las reglas - afirme que el grupo las acepta (refiriéndose entonces, desde afuera, al modo en que los participantes entienden las reglas) o según el observador se limite a registrar "las regularidades de conducta observable en que parcialmente consiste la conformidad con las reglas, y aquellas regularidades adicionales, en la forma de reacción hostil, reprobaciones o castigos, que enfrentan las desviaciones” (Hart, 1961, 111). En otras palabras: el observador externo puede tener un interés práctico o un interés teórico. Tienen un interés práctico quienes buscan adecuar su conducta a las reglas por temor al castigo o por la esperanza de obtener un beneficio, tal que sería precisamente el temor al eventual castigo o la esperanza de un eventual beneficio la razón para actuar, y no la regla. Por el contrario, tienen un interés teórico aquellos que solamente están interesados en el análisis, la explicación o la justificación de las reglas (Gaido, 2010, 152; Shapiro, 2006, 1160). Ahora bien, agrega Hart que, si el observador se atiene exclusivamente a registrar regularidades y no ofrece una explicación sobre la manera en que los miembros del grupo que aceptan las reglas contemplan su propia conducta, la descripción no será una descripción en términos de reglas. Ese punto de vista externo rígido no puede explicar la manera en que las reglas funcionan en la vida de

...los funcionarios, abogados o particulares que las usan, en situación tras situación, como guías para conducir la vida social, como fundamento para reclamaciones, demandas, reconocimientos, críticas o castigos, esto es, en todas las transacciones familiares de la vida conforme a reglas. Para ellos la viola- 
ción de una regla no es simplemente una base para la predicción de que sobrevendrá cierta reacción hostil, sino una razón para esa hostilidad (Hart, 1961, 113).

En ese sentido, Hart entiende que una de las dificultades que enfrenta una teoría que pretende dar cuenta de la naturaleza del derecho como una cuestión de reglas, es no perder de vista la existencia de ambos puntos de vista.

En la definición hecha por Hart del punto de vista interno (1961, 111-113 y 249) se mezclan un componente cognitivo y uno volitivo, de modo que se presentan como inescindibles la comprensión del seguimiento de reglas en tanto fenómeno social (no meras regularidades de conducta) y la aceptación de dichas reglas (Bayón, 1991a, 25). Sin perjuicio de ello, Hart admite que ambos componentes son separables, lo cual ha permitido incorporar al esquema de Hart, por ejemplo, los aportes de Raz.

Si la comprensión de fenómenos sociales en términos de seguimiento de reglas es separable de la aceptación de esas mismas reglas, entre los enunciados desde un punto de vista externo extremo, que se limitan a registrar regularidades de conducta, y los genuinos enunciados internos, con los que el hablante manifiesta su aceptación de una regla y hace uso de ellas como criterio de evaluación de conductas, queda espacio para un tercer tipo de enunciados (Bayón, 1991a, 27).

A dichos enunciados Raz los denomina imparciales o no comprometidos. Exhiben un uso del lenguaje normativo por el hablante, quien los formula desde el punto de vista de quien acepta la regla, pero sin aceptarla él, a su vez. La relevancia de los enunciados "no comprometidos" o "hermenéuticos" radica en que, por una parte, constituyen enunciados sobre lo que el derecho es o sobre lo que el derecho requiere para un caso y, por otra parte, quien formula tales enunciados o argumenta sobre su base lo hace adoptando el punto de vista interno, sin ser él mismo un participante, lo cual significa que no comparte sus premisas ni está vinculado por sus compromisos normativos (Colomer, 2006, 295). La particularidad de estos enunciados es que permiten emplear el lenguaje prescriptivo en la descripción de un sistema jurídico sin que por ello se asuma un compromiso valorativo (es decir, sin que sea necesaria la aceptación de las reglas). En otras palabras, estos enunciados permiten diferenciar con éxito entre la exigencia de reconocer la prioridad del punto de vista del participante y la necesidad de la aceptación de las reglas del sistema. Para iden- 
tificar una práctica social como el derecho, no es necesario comprometerse con ella uno mismo ni asumir personalmente que la práctica sea valiosa o esté moralmente justificada (Colomer, 2006, 295).

Por su parte, Bayón (1991b, 27-28) entiende que entre ambos tipos de enunciados hay diferencias, en tanto el tercer tipo de enunciado desde el punto de vista externo (Hart, 1961) es un enunciado acerca del hecho de que otros individuos aceptan reglas y actúan guiados por ellas. Los enunciados imparciales, por su parte, no son enunciados acerca de esos hechos, sino formulados desde el punto de vista de aquellos que aceptan las reglas (pero que el hablante formula hipotéticamente, sin hacerlos suyos o sin comprometerse con dichos puntos de vista, esto es, sin proferir genuinos juicios prácticos). En ese sentido, según Bayón, se aproximan más a los enunciados de deber ser en sentido descriptivo kelsenianos (Kelsen, 1960; Hart, 1983) $)^{(12)}$.

Para Hart, en definitiva, la existencia del derecho como sistema de reglas depende de que los integrantes de la práctica (de manera típica los jueces) acepten, o adopten, el punto de vista interno respecto de una práctica social consistente en reconocer competencia para ser fuente de derecho a ciertos hechos (Gaido, 2010, 151).

Quienes adoptan dicha perspectiva aceptan al derecho como constitutivo de pautas o guías de conducta que emplean como fundamento para la propia acción, así como para la crítica del comportamiento de quienes se apartan de ellas (Gaido, 2010, 151; Perry, 2006, 1172; Shapiro, 2006, 1157). Ahora bien —según Hart— el derecho es constitutivo de razones justificatorias solamente para quienes adoptan el punto de vista interno (Nino, 2014), por lo cual el derecho en sí no constituiría un sistema particular de razones para la acción, sino que "su existencia como conjunto de razones depende del dato subjetivo de su aceptación” (Gaido, 2010, 151). Raz se aparta de Hart en tanto considera que el componente subjetivo es insuficiente para afirmar la existencia de una razón para actuar y, además, considera que no es posible explicar la normatividad jurídica sin vincularla a un valor moral (la autoridad legítima). Ambas afirmaciones se siguen de otorgar privilegio conceptual al punto de vista interno (Gaido, 2010, 153). Ahora bien, la diferencia entre el punto de vista no comprometido y el del aceptante permite a Raz rechazar que los criterios de relevancia de algunos rasgos del objeto de reflexión sean, en la teoría del derecho, los mismos criterios morales y políticos del participante (Colomer, 2006, 296). Diferenciar los puntos de vista permite, entonces, distinguir la diferencia entre los inte- 
reses y criterios evaluativos del teórico y del participante. De este modo, la argumentación de Raz que refiere a la cuestión de la naturaleza del derecho en general descansa en ciertos hechos no discutidos y en consideraciones evaluativas, pero la evaluación implicada no es una evaluación moral. La argumentación refiere a ciertos aspectos del razonamiento práctico y, más en general, a lo que considera relevante para nuestra consideración de los procesos sociales que forman parte de nuestro entorno (Colomer, 2006, 296).

\subsection{El punto de vista interno en la teoría de Raz}

Siguiendo a Paula Gaido (2010 y 2011), conceder al punto de vista interno privilegio conceptual supone asumir que hay un único concepto de derecho (el nuestro en cuanto comunidad cultural) y que los participantes de las prácticas jurídicas tienen una ventaja en su acceso a este. Ello, en el entendido de que, conforme Raz, es nuestro concepto de derecho el que es necesario elucidar y, para ello, la manera en que este es entendido por quienes aceptan al derecho como razones objetivas para la acción es lo que marca la línea de su correcta comprensión. La perspectiva de la teoría del derecho, entonces, requiere la adopción del concepto de derecho asumido desde el punto de vista interno (Raz, 1995; Raz, 1996; Caracciolo, 1991; Gaido, 2010) que, adecuadamente entendido, consiste en la perspectiva de quienes aceptan la legitimidad de la pretensión de autoridad del derecho (Perry, 2006, 1201): “admitimos la prioridad del punto de vista del participante, incluso el observador, a fin de adquirir una comprensión razonable del derecho, debe concebirlo como lo haría un participante” (Raz, 2001b, 321). A su vez, Raz afirma:

...como para entender el derecho tenemos que entender la forma en que el derecho se entiende a sí mismo (esto es, la forma en que sus funcionarios y aquellos que aceptan su legitimidad lo entienden), lo tenemos que entender cómo sería entendido por la gente que lo considera éticamente justificado, al menos, en el sentido de que es éticamente correcto obedecerlo y, por tanto, lo tenemos que entender como si fuera así justificado (1996, 34-35).

Es típico de nuestras prácticas jurídicas que se persiga la imposición de deberes a terceros (aplicación de reglas), de modo que el derecho, para aquellos que participan en tales prácticas (los jueces), al tener una función justificatoria, no puede dejar de tener un fundamento moral. Ello porque, según Hart, el participante no puede aceptar — si es sincero - reglas que imponen deberes a otras personas a menos que se tengan razones morales (Raz, 1984, 130) para hacerlo. Si este es el rasgo deter- 
minante para comprender la normatividad del derecho y, por tanto, la naturaleza de su carácter justificatorio, es necesario elucidar el tipo de vínculo que se traba entre derecho y moral desde el punto de vista interno o de los participantes (Gaido, 2010, 157). Los jueces, cuando adoptan la regla de reconocimiento como guía para identificar el derecho, lo que hacen es aceptar una regla que les exige que acepten otras reglas que imponen deberes a terceros (Raz, 1984), de manera que, por razones conceptuales, los participantes deben considerar al derecho como una particular fuente de razones morales. Más precisamente: desde la perspectiva de los participantes la aceptación implica una específica creencia moral, la cual sostiene que el derecho es una estructura de autoridad moral o legítima (Gaido, 2010, 157). Se presupone, además, que esa autoridad legítima es afirmada por quienes emiten las directivas autoritativas, dada la particular naturaleza de los intereses afectados por las reglas. La autoridad legítima es entonces el valor moral al que está vinculado el derecho(13). Raz define a la autoridad de facto como una entidad que o bien pretende autoridad legítima o bien respecto de la cual existe la creencia de que tiene autoridad legítima, y que, a su vez, es efectiva en imponer su voluntad respecto de aquellos sobre quienes pretende tener autoridad, tal vez porque su pretensión de legitimidad es reconocida por un importante número de esos sujetos (Raz, 2001; Perry, 2006, 1202). Nuestros conceptos de derecho y de sistema jurídico no requieren que la efectiva posesión de autoridad legítima sea una condición necesaria de su existencia, pero sí requieren que el sistema político de que se trate tenga al menos autoridades de facto en el sentido de Raz. Es importante insistir en cuanto a que tanto la pretensión de ser una autoridad legítima como la aceptación de la legitimidad de una autoridad involucran creencias acerca de que se posee autoridad legítima y acerca de que se está sujeto a una autoridad de ese tipo (Perry, 2006, 1202; Raz, 1986b, 65).

En ese sentido, a pesar de que, conceptualmente, el derecho — desde el punto de vista interno - es fuente de razones justificatorias o de razones objetivas para actuar, no lo es necesariamente en los hechos, puesto que puede fracasar en su propósito $^{(14)}$. En ese sentido, la aceptación de las directivas de la autoridad es concebible a pesar de que la creencia efectiva en su legitimidad no esté presente.

Si por una razón conceptual la aceptación de la autoridad expresa o manifiesta la creencia en la proposición afirmada, este es el dato relevante, no la efectiva actitud psicológica que tuvo el autor de la afirmación. Lo que tiene que contar, para Raz, son los compromisos conceptuales que el aceptante adquiere, no sus contingentes componentes psicológicos (Gaido, 2010, 158). 
Bastaría el acto de usar las reglas como razones para afirmar que hay aceptación, si bien es posible conceptualmente pensar en la existencia de un sistema jurídico cuyos aceptantes no crean en su legitimidad. Puede ser innecesario para los participantes creer genuinamente que la autoridad efectiva o de facto sea la autoridad legítima; es suficiente si ellos confiesan tal creencia (Raz, 1982, 46-49; Perry, 2006, 1201-1202). Adoptar el punto de vista interno es, de alguna manera, equivalente a la creencia de que la pretensión de legitimidad alegada por una autoridad de facto está justificada (Perry, 2006, 1202).

Por otra parte, el derecho será fuente de razones objetivas para actuar solo si su autoridad es efectivamente legítima. La legitimidad de la autoridad, en cada caso, no se evalúa considerando todas y cada una de sus directivas ni tampoco teniendo en cuenta a la totalidad de los destinatarios de modo general, sino que se evalúa desde el punto de vista de cada individuo (Raz, 1986b, 73-105), razón por la cual no es posible hacer referencia a la existencia de un deber general de obediencia del derecho o de una obligación general de obedecer. De acuerdo a la interpretación de Gaido de la teoría de Raz, se afirma que "si las disposiciones jurídicas son válidas — esto es, si tienen como origen a la autoridad legítima - tienen fuerza vinculante (moral) para todos aquellos que sean sus destinatarios” (2010, 159). Así, Raz no equipara legitimidad moral con corrección moral y, por tanto, puede haber normas cuyo origen sea una autoridad legítima pero que, individualmente consideradas, sean moralmente incorrectas. Por el contrario, si la autoridad (fuente de origen) no es legítima, el carácter de razón justificatoria de la disposición puntual no se verifica. En definitiva, la normatividad del derecho es un dato eventual, que depende de la legitimidad de la autoridad.

\subsection{Privilegio conceptual y privilegio epistémico}

Es posible distinguir entre poseer un concepto y dominar un concepto. Se posee un concepto cuando se está en condiciones de identificar casos que lo ejemplifiquen, en circunstancias normales, con independencia de que las propiedades que se tengan en cuenta constituyan rasgos esenciales del objeto al cual el concepto hace referencia. Es posible que se cometan errores en la identificación porque las propiedades tenidas en cuenta solamente son útiles en circunstancias normales, pero no en cualquier ocasión. En ese sentido, es posible que el participante de la práctica jurídica pueda cometer errores en la identificación de casos concretos que ejemplifican el concepto de autoridad legítima (aceptando como autoridad legítima a una institución que carezca de tal atributo), aun poseyendo el concepto de autoridad 
legítima (Gaido, 2010, 160). Por el contrario, se domina un concepto si se tiene conocimiento de todas las propiedades necesarias o esenciales del objeto al cual hace referencia el concepto. Aun en este caso, es posible el error en la aplicación, en la medida en que en casos en los cuales el dominio del concepto es completo es posible padecer déficits pragmáticos que afecten la identificación de ejemplos correctos del concepto; con lo cual queda excluida "cualquier posibilidad de conexión necesaria entre creencia en la legitimidad de una autoridad de facto y su legitimidad efectiva” (Gaido, 2010, 160). Esta particularidad permite distinguir dos niveles de prácticas subyacentes en el análisis del concepto de derecho: una práctica jurídica y una conceptual; lo cual permite, a su vez, distinguir entre participantes de la práctica jurídica y participantes de la práctica conceptual.

En el contexto de la práctica jurídica los participantes están comprometidos con la idea de que el derecho tiene autoridad legítima. Desde esta perspectiva, negar la existencia de la autoridad legítima es incompatible con afirmar la existencia del derecho. Quienes participan de la práctica conceptual (...) podrían señalar que quienes participan en una determinada práctica jurídica están equivocados respecto de la práctica justificatoria con la cual manifiestan estar involucrados (Gaido, 2010, 161).

En definitiva, desde el punto de vista interno, la relación entre el derecho y la moral es una relación necesaria. El derecho tiene autoridad legítima desde la perspectiva del participante. Afirmar la ausencia de autoridad legítima desde esa perspectiva es afirmar la ausencia de derecho. Comprender la normatividad del derecho requiere conceder al punto de vista interno primacía lógica sobre el externo. Aclara Carlos Nino que si bien es obvio que si no hay sujetos que adopten el punto de vista interno no hay fenómeno que pueda ser observado desde la perspectiva externa es menos obvio que la observación externa no pueda desarrollarse apropiadamente sin tener en cuenta el punto de vista interno (Nino, 2014, 51) ${ }^{(15)}$. La creencia moral o política en la legitimidad del derecho, aunque sea una creencia equivocada, tiene que ser reconocible (Raz, 1997). En este sentido, el alcance de la noción de creencia moral es de carácter mínimo: "para que una creencia acerca de cómo se deben comportar otras personas sea reconocible como una creencia moral tiene que, por lo menos, aparentar ser sostenida sobre la base de una justificación que excede el autointerés” (Gaido, 2010, 162). 
Está en la naturaleza del derecho pretender o reclamar autoridad legítima sobre todos los integrantes de una comunidad política. Es decir: todo sistema jurídico pretende poseer un poder moral para exigir obediencia a los sujetos que pertenecen a una comunidad política. Para que ello sea efectivamente así, es necesario que el ordenamiento jurídico en cuestión sea capaz de tener autoridad (Marmor, 2010; 2011), para lo cual — a su vez — se requiere que los sujetos que integran la comunidad puedan identificar las directivas de la autoridad sin recurrir a consideraciones morales, es decir, sin recurrir al propio juicio moral. Ese es el rol mediador de la autoridad política, en la medida en que quien la ejerce se interpone entre los individuos y las razones que estos tienen para actuar. Las directivas de la autoridad pretenden reemplazar las razones de los sujetos mediante una directiva excluyente. En esto consiste esencialmente la concepción de la autoridad como servicio formulada por Raz (2006; Ródenas, 2006). Si los sujetos necesitaran recurrir al propio balance de razones para decidir qué deben hacer en cada caso, entonces la autoridad política no estaría actuando como tal (Perry, 2006, 1201; Raz, 2006).

No se comprende al derecho si no se lo considera moralmente inteligible, esto es, la actitud de las personas con relación al derecho es moralmente inteligible (que ellos crean que el derecho debe ser moralmente obligatorio). El concepto de autoridad legítima no asume que el derecho sea bueno ni que las creencias de las personas hacia el derecho sean sólidas, pero implica un supuesto normativo en la medida en que, por un lado, la distinción entre lo que es moralmente inteligible y lo que no lo es, es una distinción que depende de valoraciones, y, por otro, aunque el derecho sea moralmente defectuoso o carezca de toda autoridad pretende que tiene autoridad (moral) y es, por tanto, contemplado de ese modo por las personas que lo aceptan como obligatorio (Raz, 1997, 212). "Que el derecho sea moralmente inteligible se sigue del hecho de que muchas personas que viven bajo él creen que es moralmente adecuado o aceptable” (Raz, 1997, 212). Si es necesariamente el caso que la aceptabilidad moral de un sistema jurídico es una creencia de muchos de los participantes en la práctica, entonces el derecho debe ser moralmente inteligible, ya que debe ser inteligible que ellos tienen esa actitud hacia su derecho. Ello es consistente con la situación de amplias poblaciones que viven bajo el yugo de un derecho opresor hacia el cual no sienten lealtad alguna. Esto es verdad incluso si solamente un grupo de los participantes en la administración del derecho, en la dirección del gobierno, y algunos de los que se benefician de ello creen en la validez moral del derecho. Raz se pregunta por qué los involucrados en crear o aplicar el derecho deben creer en su aceptabilidad moral: porque el derecho pretende determinar o reflejar los derechos y deberes (morales) de sus sujetos (Raz, 1997, 212). En eso consiste la 
pretensión de autoridad, que es el rasgo esencial del concepto de derecho. Sostiene Gaido que es posible inferir de estas afirmaciones de Raz que la creencia moral implicada en el acto de justificar la imposición de deberes a otras personas introduce en nuestro concepto de derecho un estándar de excelencia moral que afirma que el valor intrínseco del derecho es ser legítimo (Gaido, 2010, 163). La idea de legitimidad constituiría el estándar de excelencia propio del derecho ${ }^{(16)}$. En ese sentido, "no obstante ser necesaria la idea de legitimidad para comprender la idea de derecho, la existencia de derecho no dependería de la verificación de su legitimidad” (Gaido, 2010, 163). Lo que hace a la excelencia intrínseca del derecho una excelencia moral es el hecho de que consiste en una estructura de autoridad.

Necesariamente, el derecho pretende tener autoridad legítima moral sobre sus súbditos. De ahí que su virtud intrínseca es tener tal autoridad. Decir esto es decir que su virtud es ser moral pero de un modo especial, reuniendo las condiciones de legitimidad (Raz, 2003).

Que el derecho sea efectivamente legítimo es una cuestión contingente. El rasgo necesario del derecho es que pretende ser una estructura de autoridad legítima. Teniendo en cuenta esta distinción entre la pretensión de autoridad legítima (que constituye la naturaleza del derecho) y la contingencia de que el derecho efectivamente la posea, se plantea la pregunta de si afirmar tal cosa no supone el abandono de la perspectiva del participante como punto de vista teórico. En otras palabras: aun admitiendo que cualquier persona

que tenga un mejor manejo del concepto puede constatar que no siempre el que cree que es una autoridad legítima lo es, o no siempre de quien se cree que tiene autoridad legítima la tiene, si desde el punto de vista de los participantes no hay derecho sin autoridad legítima, en los casos donde se verifique la ausencia de autoridad legítima habrá que concluir que no hay derecho (Gaido, 2010, 164).

Por ello, para poder afirmar que el rasgo necesario del derecho es la pretensión de autoridad legítima, y no la autoridad legítima efectiva, Raz abandona el compromiso metodológico de atribuirle prioridad conceptual al punto de vista interno (Gaido, 2010, 164). 
Ahora bien, el principal hallazgo de Raz en la caracterización de la autoridad se encuentra en la afirmación de que es racional actuar siguiendo la directiva de otro cuando al hacerlo el sujeto tiene más posibilidades de cumplir u obedecer las razones que se le aplican en las circunstancias relevantes. Una persona tiene autoridad sobre otra si es más probable que el sujeto que cumple con las razones que ya se le aplican (que no son las de la autoridad) acepta las directivas de la (supuesta) autoridad como vinculantes e intenta seguirlas en lugar de intentar él mismo seguir las razones que se le aplican directamente. En esta afirmación es que se basa la tesis de la justificación normal, es decir, sobre la racionalidad del acatamiento de las directivas de la autoridad cuando el sujeto — siguiendo la directiva - cumple mejor con las razones que ya se le aplican que si intentara seguir esas razones directamente ${ }^{(17)}$. Por eso se entiende que las autoridades existen para proveer un servicio: el de hacer que los sujetos actúen por las razones adecuadas que se les aplican sin tener que entender por sí mismos esas razones o actuar directamente por ellas (Marmor, 2010 , 4). Lo anterior se vincula a la naturaleza de reemplazo (preemptive) que poseen las razones protegidas ${ }^{(18)}$. Que una directiva autoritativa exija realizar una acción es una razón para actuar en correspondencia; es una razón que no se debe agregar a otras razones relevantes al momento de determinar qué se debe hacer. Esas razones, que son producto de la directiva, reemplazan a las otras razones.

\subsection{Razones para la acción}

La de Raz es (a diferencia de Hart) una versión objetivista de las razones para actuar $^{(19)}$, en la medida en que estas existen como hechos independientes de las creencias, actitudes, disposiciones y comportamientos efectivos de los individuos para quienes son razones para la acción. Las razones se aplican a esos individuos y estos las cumplen cuando realizan el acto requerido, aun cuando no lo hayan determinado subjetivamente (Caracciolo, 1991, 78). Como las normas válidas pertenecen a la clase de las razones excluyentes, su existencia es una cuestión objetiva, aun cuando no existan como prácticas de comportamiento. "Inversamente, los que aceptan que ciertas prescripciones son normas válidas, tienen que creer que constituyen razones objetivas para actuar” (Caracciolo, 1991, 79). Pero la validez es distinta de la creencia en la validez. En la medida en que el deber supone restricciones al comportamiento de los destinatarios, las razones objetivas para justificar la autoridad no pueden basarse en el autointerés de los sujetos que la constituyen. De acuerdo al sentido genuino de validez y de deber, semejantes razones tienen que ser razones morales, que se imponen más allá de los intereses y preferencias individuales (Raz, 1984). Quienes pretenden autoridad sinceramente y quienes la reconocen 
creen que sus enunciados internos son verdaderos. Creen que la autoridad es legítima (Caracciolo, 1991, 79).

Para la versión objetivista de Raz, la creencia en las razones morales define la condición de aceptante (participante) en tanto que los enunciados internos afirman la existencia de razones objetivas. Si se admite esta tesis — sostiene Ricardo Caracciolo - "es coherente sostener que el punto de vista interno es una alternativa al punto de vista externo: mientras los enunciados externos se refieren a conductas y actitudes subjetivas, los internos versan sobre razones objetivas” (1991, 80). Por tanto, la primacía o el privilegio conceptual indican que es preferible una noción comprometida de autoridad para comprender lo que significa pretender y reconocer autoridad.

\section{Normas jurídicas como razones excluyentes}

No todas las manifestaciones autoritativas son normas, pero dado que todas las manifestaciones autoritativas son razones excluyentes ${ }^{(20)}$, las normas dictadas por una autoridad son razones excluyentes. Las razones excluyentes son — como señalé en apartados anteriores- "razones para no actuar por ciertas razones” (Raz, 1991, 226). La caracterización del derecho elaborada por Raz se conforma con el análisis de su noción de autoridad legítima, la cual —a su vez - no resulta inteligible si no se tiene presente la noción de razón excluyente (que Raz sitúa como un elemento clave en su reconstrucción de la estructura del razonamiento práctico) (Bayón, 1991b, 25).

El propósito de la teoría de las normas como razones para la acción es mostrar que si la autoridad ha de justificarse por las exigencias de la coordinación, es necesario considerar a sus manifestaciones como razones excluyentes. Ello, porque la autoridad puede asegurar la coordinación solamente si los ciudadanos afectados dejan de lado su juicio y, en lugar de actuar sobre la base del balance de razones, lo hacen sobre la base de las instrucciones de la autoridad. Considerar a alguien como una autoridad implica considerar a algunas de sus manifestaciones como autoritativas, aunque puedan ser incorrectas sobre la base del balance de razones. En eso consiste considerar a una manifestación autoritativa como una razón excluyente (Raz, 1991, 73). En otras palabras, suele aceptarse que el reconocimiento de una autoridad como legítima implica aceptar su derecho a mandarme y mi deber de obedecerle (Bayón, 1991b, 26), a la vez que ese reconocimiento implica que una directiva de la autoridad no es solo una razón para comportarse como ella ordena, 
sino también una razón para no seguir razones que chocan con la directiva ${ }^{(21)}$ (Raz, 2013, 155).

Las oraciones acerca de la existencia de normas se usan, fundamentalmente, con tres propósitos, los cuales son decir que una norma es válida (esto es, justificada), que la norma es practicada (esto es, eficaz) o bien que ha sido prescrita por una cierta persona u órgano. Expondré a continuación con más detalles estos propósitos.

En primer lugar, una norma es válida si, y solo si, los sujetos normativos están justificados en guiar su conducta por ella siempre que ella sea aplicable; es decir, siempre que se den sus condiciones de aplicación. Ahora bien, la cuestión de la validez se distingue de otras cuestiones de justificación, en la medida en que una norma puede ser válida y sin embargo su sujeto normativo puede no estar justificado en realizar el acto normativo en ciertas circunstancias (porque pueden estar presentes otras razones en conflicto no excluidas por la norma, que deben prevalecer) (Raz, 1991, 91).

Agrega Raz que una norma legislada puede ser válida aunque la autoridad legislativa no estuviera justificada al dictarla. Sin embargo, una vez dictada, los sujetos normativos tienen razones para considerar a esa norma como vinculante a pesar del hecho de que no debería haber sido dictada. En definitiva, cuando se hace referencia a la validez de las normas se está aislando un problema de justificación, que es: ¿deben los sujetos guiar su conducta por la norma? En segundo lugar, una norma válida puede ser practicada o no. Que sea practicada implica que al menos algunos creen que es válida, pero de ninguna manera esto implica que lo sea ${ }^{(22)}$. En tercer lugar, una norma es prescrita si es establecida por un individuo o grupo como una norma para guiar la conducta de terceros.

Una norma prescrita es una norma establecida por un acto de habla que pretende ser tomado por los sujetos normativos como una razón excluyente para actuar (Raz, 1991, 93). Cuando se afirma la existencia de normas, en definitiva, la afirmación implica que estas poseen una o más de estas propiedades (válidas, practicadas, prescritas). De las tres dimensiones, la de validez es sin duda la más relevante: solo las normas válidas son razones válidas (o buenas) (Raz, 1991, 95-96).

La presencia de órganos primarios — que caracteriza a un genuino sistema normativo como lo es el sistema jurídico - supone el funcionamiento combinado de dos tipos de relaciones de autoridad. Por un lado, tales órganos resuelven conflictos 
con arreglo a criterios provenientes de fuentes a las que consideran dotadas de autoridad(23) $^{(23}$, por otro lado, son considerados a su vez como autoridades por otros órganos $^{(24)}$ (Bayón, 1991b, 33).

\section{Sistemas institucionalizados. Instituciones aplicadoras de normas}

\subsection{Características}

Los sistemas institucionalizados son conjuntos de normas que o establecen ciertas instituciones aplicadoras de normas o están internamente relacionados con ellas de alguna manera. Para comprender la naturaleza de los sistemas institucionalizados abordaré el tema de en qué consisten las instituciones aplicadoras de normas y cuáles son las relaciones entre las normas que crean a dichas instituciones y el resto de las normas del sistema (Raz, 1991, 151-152). En primer lugar, me ocuparé de analizar cuáles son los rasgos distintivos de los órganos de aplicación de normas, deteniéndome específicamente en las instituciones que Raz llama órganos primarios: "instituciones con poder para determinar la situación normativa de individuos concretos, a los cuales se exige que ejerzan esos poderes aplicando normas existentes, pero cuyas decisiones son vinculantes aun cuando son incorrectas” (Raz, 1991, 156). Es decir, se trata de una clase de instituciones que contienen normas que confieren poderes a órganos centralizados para hacer determinaciones aplicativas vinculantes. Los tribunales y otros órganos judiciales son el ejemplo más importante de órganos primarios.

Afirmar que la presencia de un órgano primario es un rasgo definitorio de los sistemas institucionalizados se basa, sostiene Raz, en el conocimiento común de casos típicos de sistemas jurídicos y otros sistemas semejantes y, fundamentalmente, en el especial papel que dichas instituciones juegan, cuando existen, en la regulación de las relaciones sociales. "La presencia de instituciones primarias indica que el sistema normativo de que se trate proporciona una manera institucionalizada y autoritativa de resolver las disputas.” (Raz, 1991, 158)

\subsection{Los sistemas institucionalizados y las razones excluyentes: a modo de conclusión}

Precisamente, la importancia de las instituciones primarias radica en que proveen un método autoritativo institucionalizado de resolver las disputas. Además, tienen el deber de aplicar reglas existentes. Es decir que los órganos primarios aña- 
den al sistema la dimensión de las valoraciones autoritativas de la conducta, valoración que se realiza sobre la base de los estándares que los órganos primarios del sistema están obligados o vinculados a aplicar.

Los sistemas institucionalizados —al contrario de los sistemas de discreción ab-

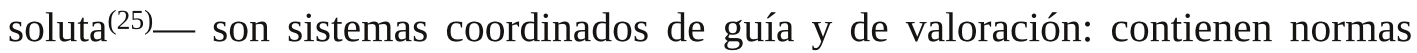
que guían la conducta y, a su vez, contienen instituciones para valorarla y juzgarla (Raz, 1991, 160). A su vez, los sistemas institucionalizados se componen de normas que los tribunales están vinculados a aplicar con independencia de su opinión sobre su mérito. Se componen de normas que los órganos primarios del sistema no están en libertad de no tomar en consideración. "Les está permitido actuar sobre la base de sus propias opiniones solo hasta el punto en que ello está permitido por aquellas normas."(26) (Raz, 1991, 160) Los estándares sobre cuya base deben actuar los órganos primarios son las reglas del sistema bajo el que operan, y deben excluir los estándares que no forman parte del sistema; esto es: los órganos primarios deben actuar sobre la base de ciertas razones con exclusión de todas las demás. En otras palabras, están sujetos a una razón excluyente para no actuar sobre la base de ciertas razones (Raz, 1991, 164).

Los tribunales juzgan a los individuos sobre la base de las reglas jurídicas, excluyendo las demás consideraciones. Por consiguiente, deben juzgar a los individuos como si estos debieran tomar a los estándares jurídicos como razones excluyentes. Esto, porque las reglas del sistema que se aplican a los individuos son idénticas a las reglas mediante las cuales los órganos primarios deben juzgar la conducta de los individuos (Raz, 1991, 166).

En ese sentido, a partir de la distinción entre reglas jurídicas de mandato, permisivas y que confieren poderes (Raz, 1986a; Raz, 1991), Raz especifica: 1) toda regla jurídica que exija la realización de una acción (u omisión) es una razón para la realización (u omisión) de esa acción y también una razón excluyente para no actuar sobre la base de razones en conflicto que no sean ellas mismas normas jurídicas o razones jurídicamente reconocidas; 2) toda regla jurídica permisiva es también una permisión excluyente, es decir, una permisión para realizar el acto normativo y una permisión de segundo orden para no actuar sobre la base de razones para no realizar el acto normativo que no sean ellas mismas normas jurídicas o razones jurídicamente reconocidas; 3) toda regla jurídica que confiera poderes está relacionada con normas jurídicas de mandato y normas jurídicas permisivas del siguiente modo: se asemejan a las normas permisivas y difieren de las de mandato en que tienen una 
fuerza normativa, sin ser ellas mismas razones completas para la acción.

Su fuerza normativa se manifiesta en que los enunciados de las normas que confieren poderes son premisas de inferencias prácticas que afectan a la conclusión de la inferencia. Al considerar si se realiza una acción, el hecho de que tenga ciertas consecuencias normativas es una consideración relevante. No es ella misma una razón para realizar la acción o para abstenerse de realizarla (...) Pero, como las normas permisivas, las normas que confieren poderes son normativas y puede decirse que guían la conducta a causa de su contribución a inferencias prácticas, a la solución de problemas prácticos (Raz, 1991, 120).

En definitiva, un sistema normativo que contenga normas que guíen a los individuos y normas que establezcan instituciones para resolver las disputas surgidas de la aplicación de las primeras; esto es, un sistema basado en órganos primarios, es necesariamente un sistema excluyente (sus normas excluyen la aplicación de razones que no pertenezcan al sistema o que no sean reconocidas por el sistema). El punto de vista jurídico es un punto de vista excluyente: puede haber normas jurídicas en conflicto y al decidir qué debe hacerse puede corresponder ponderar consideraciones jurídicas en conflicto, pero el derecho es un sistema excluyente y excluye la aplicación de razones extrajurídicas (Raz, 1991, 167). 


\section{Referencias}

Bayón, J. C. (2002). Derecho, convencionalismo y controversia. En P. Navarro y M. C. Redondo (comps.). La relevancia del derecho. Ensayos de filosofía jurídica, moral y política (pp. 57-92). Barcelona: Gedisa.

Bayón, J. C. (1991a). La normatividad del derecho. Deber jurídico y razones para la acción. Madrid: Centro de Estudios Constitucionales.

Bayón, J. C. (1991b). Razones y reglas: sobre el concepto de 'razón excluyente' de Joseph Raz. Doxa, (10), 25-66.

Bulygin, E. (1981). Enunciados jurídicos y positivismo: respuesta a Raz. Análisis Filosófico, 1(2), 49-59.

Caracciolo, R. (1991). El concepto de autoridad normativa. El modelo de las razones para la acción. Doxa, (10), 67-90. Recuperado de https://rua.ua.es/dspace/ bitstream/10045/10761/1/doxa10_02.pdf

Coleman, J. (ed.) (2001). Hart's Postscript. Essays on the Postscript to the Concept of Law. Nueva York: Oxford University Press.

Colomer, J. L. (2006). Teoría del Derecho, interpretación y punto de vista interno: algunas observaciones sobre las tesis de Ronald Dworkin. En J. Ramos Pascua y M. Rodilla González (Eds.). El positivismo jurídico a examen: Estudios en homenaje a José Delgado Pinto (pp. 277-302). Salamanca: Universidad de Salamanca.

Gaido, P. (2010). El privilegio conceptual de los participantes en la teoría del derecho de Joseph Raz. Isonomía, (32), 149-164. Recuperado de http://www.scielo .org.mx/scielo.php?script=sci_arttext\&pid=S1405-02182010000100007

Gaido, P. (2011). Las pretensiones normativas del derecho. Un análisis de las concepciones de Robert Alexy y Joseph Raz. Madrid: Marcial Pons.

Hart, H. L. A. (1961). El concepto de Derecho. Buenos Aires: Abeledo Perrot.

Hart, H. L. A. (1980). El nuevo desafío al positivismo jurídico. Sistema, (36), 3-18. 
Hart, H. L. A. (1983). Kelsen visited. Essays on Jurisprudence and Philosophy, 287295.

Holmes, O. W. (1897). The Path of the Law. Harvard Law Review (1996-1997), 10, 457.

Kelsen, H. (1960). Teoría pura del Derecho. Ciudad de México: UNAM.

Marmor, A. (2010). The Dilemma of Authority. University of Southern California Legal Studies Papers, 10-6. Recuperado de https://papers.ssrn.com/sol3/papers .cfm?abstract_id=1593191

Marmor, A. (2011). An Institutional Conception of Authority. Philosophy \& Public Affairs, 39(3), 238-261.

Nino, C. S. (2014). Derecho, moral y política. Una revisión general de la teoría del Derecho. Buenos Aires: Siglo Veintiuno.

Orunesu, C. (2007). Sobre la inconsistencia teórica del positivismo incluyente. Análisis filosófico, 27(1), 23-46.

Perry, S. (2006). Hart on Social Rules and the Foundations of Law: Liberating the Internal Point of View. Fordham Law Review, 75, 1171-1209.

Raz, J. (1981). The Purity of the Pure Theory. Revue Internationale de Philosophie, (35), 441-459.

Raz, J. (1982). La autoridad del derecho. Ensayos sobre Derecho y moral. Ciudad de México: Ediciones Coyoacán.

Raz, J. (1984). Hart on Moral Rights and Legal Duties. Oxford Journal of Legal Studies, 4(1), 123-131.

Raz, J. (1986a). El concepto de sistema jurídico. Una introducción a la teoría del sistema jurídico. Ciudad de México: UNAM.

Raz, J. (1986b). The Morality of Freedom. Oxford: Clarendon Press. 
Raz, J. (1991). Razón práctica y normas. Madrid: Centro de Estudios Constitucionales.

Raz, J. (1995). El problema de la naturaleza del Derecho. Isonomía (3), 131-151.

Raz, J. (1996). ¿¿Por qué interpretar? Isonomía, (5), 25-40

Raz, J. (1996); Why Interpret? Ratio Juris, (9), 349-363.

Raz, J. (1997). La intención en la interpretación. Doxa (20), 199-233

Raz, J. (2001). La ética en el ámbito público. Barcelona: Gedisa.

Raz, J. (1994). Ethics in the Public Domain: essays in the morality of law a politics. Oxford: Clarendon Press.

Raz, J. (2003). About Morality and the Nature of Law. The American Journal of Jurisprudence, 48(1), 1-15.

Raz, J. (2006). El problema de la autoridad: de nuevo sobre la concepción de la autoridad como servicio. Doxa, (29), 141-175. Recuperado de https://scholar. google.com/scholar_url?url=http://www.cervantesvirtual.com/research/elproblema-de-la-autoridad--de-nuevo-sobre-la-concepcin-de-la-autoridadcomo-servicio-0/0214eed0-82b2-11df-acc7-002185ce6064.pdf\&hl=en\&sa= T\&oi=gsb-ggp\&ct=res\&cd=0\&d=14319994168206363804\&ei=ggQcXZv YFIihmQGcl6LYDg\&scisig=AAGBfm2PA19vbtL9JSRrP1cclNiQdoDAHQ

Raz, J. (2013). Entre la autoridad y la interpretación. Sobre la teoría del derecho y la razón práctica. Madrid: Marcial Pons.

Ródenas, Á. (2006). La concepción de la autoridad como servicio puesta a prueba. Doxa (29), 177-193. Recuperado de https://scholar.google.com/scholar_url?url =http://rua.ua.es/dspace/bitstream/10045/9963/1/Doxa_29_10.pdf\&hl=en\&sa= T\&oi=gsb-gga\&ct=res\&cd=0\&d=6504459302411708975\&ei=5gQcXb6TH r6Cy9YP9vK70AY\&scisig=AAGBfm2M1N-evhePnVgV--aotJlqJD4_Bg

Searle, J. (1995). The Construction of Social Reality. Nueva York: The Free Press. 
Shapiro, S. (2002). Authority. En J. Coleman y S. Shapiro (Eds.). The Oxford Handbook of Jurisprudence and Philosophy of Law (382-439). Oxford: Oxford University Press.

Shapiro, S. (2006). What Is the Internal Point of View? Fordham Law Review, 75, 1157-1170.

Vernengo, R. (1986). Kelsen's Rechtssatze as detached statements. En R. Tur y W. Twining (Eds.). Essays on Kelsen (pp. 99-108). Oxford: Clarendon Press.

Vilajosana, J. (2007). Identificación y justificación del derecho. Madrid: Marcial Pons.

Waluchow, W. (2007). Positivismo jurídico incluyente. Madrid: Marcial Pons.

\section{Notas}

${ }^{1}$ La existencia de un hábito reposa en la existencia de una conducta recurrente. En cambio, la existencia de una regla requiere, además de la regularidad de la conducta, de la convicción de la obligatoriedad de esa conducta. Se exige, señala Vilajosana, una "actitud crítico-reflexiva, que se manifiesta en la conciencia de que esa conducta que se sigue es de obligado cumplimiento y en las críticas a quienes no la realizan” $(2007,33)$.

${ }^{2}$ En el tratamiento de la dimensión constitutiva de las convenciones, Vilajosana (2007, 44-45) propone recurrir a la distinción entre reglas regulativas y constitutivas. Las primeras prohíben $\mathrm{u}$ obligan a una determinada conducta. Las segundas contribuyen a la creación de la realidad social y su fórmula canónica es " $\mathrm{X}$ cuenta cómo Y en el contexto C" (Searle, 1995). Propone considerar a la regla de reconocimiento como una regla o convención constitutiva, ya que lo que cuenta como derecho en una determinada comunidad proviene del uso de determinados criterios de identificación del derecho compartidos por los juristas (Vilajosana, 2007, 44).

${ }^{3} \mathrm{La}$ existencia de los hechos naturales es independiente de cualquier estado intencional. Por el contrario, los hechos sociales existen solo si existen ciertos estados intencionales, como creencias, deseos o actitudes (Vilajosana, 2007, 34). 
${ }^{4}$ Recordemos que autores como Waluchow (2007) y Coleman (2001) proponen como rasgo común del positivismo jurídico la tesis convencionalista: el derecho es una función de prácticas sociales. Indicar cuáles son los criterios que determinan qué normas son parte del derecho es una cuestión enteramente convencional. Ello remite al carácter social de la regla de reconocimiento, lo cual, según los mencionados autores, salvaguarda la tesis de las fuentes sociales, cuya verdad es contingente, en tanto la verdad de la tesis convencionalista es necesaria (pues, tanto pueden existir como no sistemas jurídicos que acepten que las prácticas incorporen las convenciones que reconozcan una norma como derecho en virtud de su valor o contenido). Juan Carlos Bayón (2002, 66-67) objeta, con referencia al argumento de Waluchow y Coleman, que la pretensión de aceptar la incorporación de criterios materiales de validez jurídica no es sostenible en tanto no parece posible defender la idea de la existencia de una convención de seguir criterios no convencionales (Orunesu, 2007). Bayón entiende que una regla convencional solo existe en tanto exista una práctica convergente y, por tanto, algún grado de acuerdo.

${ }^{5}$ Se discute en la literatura quiénes son los individuos cuyo comportamiento es relevante en la determinación de la regla de reconocimiento. No creemos necesario presentar esa discusión en este trabajo. En todo caso, la regla de reconocimiento es una práctica compartida entre, por lo menos, los jueces y otras autoridades.

${ }^{6}$ En efecto, el problema de coordinación que resuelve la regla de reconocimiento tiene que ver con el establecimiento de los criterios de identificación del derecho, es decir, de criterios para saber cuál es el derecho en una determinada comunidad. Determinar cuáles son esos criterios en cada caso es una cuestión vinculada a la historia institucional de cada comunidad (Vilajosana, 2007, 47).

${ }^{7}$ La unidad del sistema no depende de que contenga una sola regla de reconocimiento sino del hecho de que contiene solamente reglas que los órganos primarios están vinculados a aplicar. A su vez, los órganos primarios que han de considerarse pertenecientes a un mismo sistema son aquellos que se reconocen mutuamente el carácter autoritativo de sus determinaciones aplicativas (Raz, 1991, 169).

${ }^{8}$ Hart sostiene que los diferentes criterios de validez deben estar ordenados jerárquicamente para impedir la posibilidad de conflictos entre reglas igualmente válidas. 
${ }^{9}$ Un anarquista que sea juez, puede hacerlo en el entendido de que si sigue el derecho la mayor parte del tiempo, podrá desobedecerlo en algunas ocasiones en las cuales la desobediencia contribuya a socavarlo. Otro sujeto puede llegar a ser juez porque considera que al aplicar normas que desaprueba, si hace un buen uso de los poderes que tienen los jueces podrá hacer nuevas normas y cambiar las existentes de cuando en cuando (Raz, 1991, 170).

${ }^{10}$ Los sistemas jurídicos como sistemas institucionales cuentan con tres tipos principales de órganos. Unos son los encargados de crear (modificar, derogar) el derecho expresado en normas generales (legisladores en sentido amplio). Otros son los competentes para ejecutar físicamente la coacción (órganos ejecutivos, policiales). Otros son los que tienen competencia para qué normas son aplicables a situaciones particulares y de disponer su cumplimiento efectivo (jueces en sentido amplio). Estos últimos son los que Raz denomina órganos primarios.

${ }^{11}$ Sea porque se trata del bad man al que refiere Holmes (1897) y adopta el punto de vista práctico pero no acepta las reglas, sea porque se trata de un observador. El punto de vista teórico, a su vez, puede consistir en el punto de vista hermenéutico (Hart) o en el punto de vista externo extremo (conductista).

12 La proximidad del significado con los enunciados de deber ser en sentido descriptivo a los que hace referencia Kelsen es planteada por Raz (1981) y puesta en duda por Bulygin $(1981,56)$ y por Vernengo (1986). Nos remitimos a lo expuesto por Bayón al respecto (1991b, 28-29).

${ }^{13}$ Privilegiando el punto de vista interno para la elucidación del concepto de derecho, la pretensión de autoridad legítima determina que para los participantes el derecho es fuente de razones justificatorias (aunque en los hechos no lo sea). Los participantes, entonces, poseen un concepto de derecho que incluye un valor moral. En otras palabras, la clave para comprender en qué sentido el derecho es normativo se encuentra en el privilegio conceptual otorgado a la perspectiva del participante.

${ }^{14}$ La distinción entre autoridad legítima y autoridad de facto está relacionada con esta afirmación. La autoridad de facto pretende ser legítima (o alega serlo, o es considerada así) y es eficaz en la imposición de su voluntad sobre una cantidad de personas. No obstante, no necesariamente tiene legitimidad (Raz, 2001, 228). 
${ }^{15}$ Aun si empleamos un concepto puramente descriptivo de derecho, que sería el tipo que habilita una perspectiva externa (a diferencia de un concepto puramente normativo), no es posible que la perspectiva teórica se desentienda del punto de vista interno de los participantes en el desarrollo del derecho como institución social. Esa perspectiva interna, agrega Nino, está indisolublemente ligada a la perspectiva interna de la moral y, en especial, a la perspectiva interna de la práctica discursiva que la modernidad ha acoplado a la moral positiva (la cual tiene que ver con la dimensión justificatoria del derecho) (Nino, 2014, 53-54). Según Nino, el discurso jurídico interno no es un discurso insular sino que forma parte de un discurso justificatorio más amplio y complejo, en el cual son empleadas nociones normativas como la propia noción de derecho y la de validez jurídica $(2014,195)$. El derecho, por una parte, "visto desde la perspectiva interna está esencialmente conectado a ciertos principios ideales de justicia y moralidad social, que constituyen la fuente de las razones justificatorias en el discurso jurídico” (Nino, 2014, 196), pero, por otra, dichos principios de justicia "no se aplican directamente a las acciones o decisiones jurídicas, sino que se aplican a prácticas colectivas, de modo que si estas resultan justificadas por aquellos, las acciones y decisiones tienen que ser, por un lado, compatibles con la preservación de tales prácticas y, por otro, tienen que contribuir a que la práctica se acerque lo más posible a dichos principios ideales de justicia y moralidad" (Nino, 2014, 196). Finalmente, resulta que esos principios, "por lo menos en cuanto a su carácter intersubjetivo, no se conocen de un modo suficientemente fiable, por la mera reflexión individual aislada, sino a través de procesos colectivos de carácter democrático" (Nino, 2014, 196). De acuerdo con la opinión de Nino, en la medida en que el discurso jurídico interno forma parte de un discurso justificatorio más amplio, permite la apertura a todos de los procesos de discusión. En el entendido de que - condicionado a ciertos presupuestos y acotado a ciertas materias- "el proceso democrático tiene una tendencia general, aunque de valor solo comparativo (...) hacia soluciones moralmente correctas, el desarrollo de ese proceso refuerza la vigencia de principios ideales de justicia (...) [y esa vigencia de los principios] contribuye a que se satisfagan en mayor grado las precondiciones que determinan el valor epistémico del proceso democrático" (Nino, 2014, 197). De alguna manera, estas reflexiones de Nino pueden ser vinculadas a la crítica que ha recibido la concepción de la autoridad raziana por parte de Scott Shapiro (2002, 431-432) que, si bien defiende el positivismo jurídico excluyente de Raz, entiende cree que su concepción de la autoridad fracasa porque se centra casi exclusivamente en el valor instrumental del derecho. Por el contrario, para Shapiro, el valor de un derecho democrático no está en su valor instrumental, en tanto la democracia tiene un valor intrínseco como procedimiento de decisión colectiva. El derecho resultante de un proceso de ese tipo 
está en condiciones de reclamar obediencia. La desobediencia a las directivas autoritativas que son expresión de la voluntad democrática sería como intentar establecer unilateralmente los términos de la cooperación social (Shapiro, 2002, 437438). En sentido similar se expresa Marmor acerca de las condiciones de legitimidad de la autoridad cuando alude a la función y los objetivos de la práctica institucional, así como a que los sujetos tengan buenas razones para cooperar con la práctica (Marmor, 2011, 14).

${ }^{16}$ Andrei Marmor entiende que la legitimidad de la autoridad está conectada con el tipo de práctica de que se trate y los términos en los cuales se participa en dicha práctica, es decir, si se trata de una práctica en la que se participa voluntariamente o no (Marmor, 2010; 2011). De acuerdo con su concepción institucional de la autoridad, la legitimidad de las autoridades prácticas depende especialmente de la naturaleza de la institución en la cual la autoridad opera y de la forma de participación en dicha institución. Lo anterior opera como un límite sobre lo que puede generalizarse acerca de las condiciones de legitimidad de las autoridades, en la medida en que las prácticas y las instituciones varían considerablemente en diferentes aspectos y dimensiones y aun las que pueden considerarse buenas prácticas pueden presentar aspectos problemáticos que influyen en la legitimidad de algunas relaciones autoritativas al interior de esas prácticas. Es imposible —entiende Marmor - generalizar las condiciones que hacen a las autoridades legítimas. Existen demasiados aspectos que influyen en esta cuestión en los casos particulares. En ese sentido, afirma que nuestras razones para cooperar con instituciones o prácticas de diversos tipos son tan variadas y sensibles al contexto que no permiten contar con una fórmula general que sea aplicable a todos los casos (Marmor, 2011, 17-18).

${ }^{17}$ En este punto corresponde tener presente el contenido de la llamada tesis de la dependencia, ya que es pertinente plantearse si puede la autoridad expedir cualquier tipo de directiva $y$, por tanto, si puede exigir una conducta a los sujetos independientemente de su punto de vista. La tesis de la dependencia tiene como principal objetivo que las directivas de las autoridades se basen en razones aplicables a los sujetos, es decir, en circunstancias cubiertas por aquellas. Las autoridades serán legítimas y sus directivas constituirán razones para actuar en la medida en que tales directivas se basen en razones que sean aplicables a los sujetos. No puede la autoridad —o podrá sin ser legítima— expedir cualquier directiva que le parezca.

${ }^{18}$ El derecho posee autoridad legítima solo cuando los individuos tienen más seguridad de actuar conforme a las razones correctas si obedecen las normas jurídicas 
que si intentan actuar conforme a dichas razones directamente. Si se cumple la condición de legitimidad, los individuos pueden aceptar la autoridad política sin que haya menoscabo de su autonomía como seres racionales. A esta condición Raz agrega la denominada "cláusula de la autonomía", según la cual el Estado solo puede tener autoridad legítima sobre aquellas materias en las que es más importante actuar de acuerdo con las razones correctas que decidir por uno mismo (Raz, 2001).

${ }^{19}$ Para abordar la caracterización de Raz de las reglas conviene tener presente que, en términos históricos, en el afán teórico de determinar los rasgos de las reglas que permitan distinguirlas de otras normas que no son reglas, podemos distinguir la teoría imperativa, la teoría de la práctica y, contemporáneamente, la teoría de las razones para la acción. La teoría imperativa (Austin) de las normas prestaba atención al modo de origen y las caracterizaba como imperativos establecidos por un individuo o grupo con la intención de guiar la conducta. La teoría de la práctica tiene como representante a Herbert Hart y, básicamente, sostiene que en toda sociedad compleja existe una regla de reconocimiento que permite identificar las reglas sociales (y, por ende, las jurídicas) del grupo por medio de los criterios especificados en ella (Hart, 1961, 137). Dicha regla de reconocimiento, una vez aceptada, impone un deber a los jueces y demás funcionarios que consiste en considerar ciertos criterios específicos como identificadores de los estándares jurídicos que deben aplicar en sus decisiones (Hart, 1980, 7).

${ }^{20}$ Son también razones excluyentes las promesas, las órdenes y las decisiones (Bayón, 1991b, 41; Raz, 1991, 78-79).

${ }^{21}$ En esto consiste la noción de reemplazo que retomaremos en la tercera parte del texto.

${ }^{22}$ Según Raz, esta es una de las razones del fracaso de la teoría de las normas como prácticas, en la medida en que es un error pensar que explicando lo que es que una norma sea practicada se explica lo que es una norma (1991, 92-93).

${ }^{23}$ Es decir que los jueces aceptan una justificación para no fundar sus decisiones en el balance de razones sino en las directivas de las autoridades legislativas (Bayón, 1991b, 34).

24 “... los órganos ejecutores aceptan una justificación para un doble reemplazo: de las razones de orden general aplicables al caso por las directivas de las autoridades 
legisladoras; y de su juicio acerca de lo que exigen estas por la decisión al respecto de los órganos primarios” (Bayón, 1991b, 34).

${ }^{25} \mathrm{~A}$ los tribunales de un sistema de discreción absoluta no se les exige que decidan sobre la base de normas especificadas, por lo que no constituyen órganos primarios. La característica definitoria de un sistema de discreción absoluta es que sus tribunales están sujetos a una sola directiva para tomar sus decisiones: han de tomar siempre la decisión que consideren que es la mejor, sobre la base de todas las razones válidas (Raz, 1991, 159).

${ }^{26}$ En muchos casos, el derecho exige que los tribunales decidan en casos para los cuales el conjunto de normas al cual están vinculados no proporciona una respuesta correcta, a causa de la vaguedad, textura abierta o incompletitud. O bien, el sistema excluye determinadas soluciones como incorrectas. Si el sistema exige que los tribunales no se nieguen a resolver la disputa en estos casos, entonces se exige que determinen el caso de acuerdo con sus propias concepciones de lo correcto (Raz, 1991, 161). 